\title{
A ONE-DIMENSIONAL RANDOM WALK WITH REPULSION
}

\author{
D. F. HINES and C. J. THOMPSON
}

(Received 4 April 1978)

\begin{abstract}
A one-sided one-dimensional random walk with repulsion from the origin is solved exactly. The walk imitates the self-avoiding walk problem insofar as the mean end-to-end distance of an $n$-step walk tends asymptotically to $n$ as $n$ tends to infinity.
\end{abstract}

\section{Introduction}

Consider a walker on a regular lattice with equal step probability on bonds, but constrained so that lattice points or vertices cannot be visited more than once. This is the self-avoiding random walk problem, one of the many celebrated unsolved problems in lattice statistics on lattices with dimensionality greater than unity.

In one dimension the problem is supertrivial, since the entire walk is determined from the first step. The mean-square end-to-end distance, $R_{n}^{2}$, of an $n$-step walk, for example, is precisely $n^{2}$ in one dimension. In two and three dimensions, various heuristic [2, 3, 4], enumeration [1] and simulation [5] methods suggest the asymptotic forms $R_{n}^{2} \sim n^{3 / 2}$ and $R_{n}^{2} \sim^{6 / 5}$ respectively, while in four and higher dimensions it is generally believed that the self-avoiding walker behaves in the same way as an ordinary random walker with $R_{n}^{2} \sim n$.

A standard variational approach to the problem [4], which gives the above results, consists of replacing the hard pairwise repulsive potential by an effective single-particle repulsive potential that is determined self-consistently.

Here we consider a one-dimensional random walk with a particular singleparticle repulsive potential that can be solved exactly. We find that this walk imitates the self-avoiding walk insofar as $R_{n}^{2} \sim n^{2}$. We have not yet succeeded in constructing a generalization of this model to imitate the suspected behaviour of higher-dimensional self-avoiding walkers. 
2. The model and statement of results

Consider a one-dimensional lattice of points, $r=0,1,2, \ldots$, and a walker starting at the origin, with step probability from $r$ to $r^{\prime}$ given by

$$
p\left(r, r^{\prime}\right)= \begin{cases}\frac{1}{r+1} & \text { if } r^{\prime}=r-1 \geqslant 0, \\ i-\frac{1}{r+1} & \text { if } r^{\prime}=r+1 \geqslant 2, \\ 1 & \text { if } r^{\prime}=r+1=1, \\ 0 & \text { otherwise. }\end{cases}
$$

In this example, the origin is a reflecting barrier and it is clear that the walker is repelled from the origin with increasing $r$.

Let $P_{n}(r)$ denote the probability that the walker is at point $r$ after $n$ steps. Then from (2.1), we have for $n>0$,

and

$$
\left.\begin{array}{l}
P_{n}(r)=\left(1-\frac{1}{r}\right) P_{n-1}(r-1)+\frac{1}{r+2} P_{n-1}(r+1) \text { if } r \geqslant 2, \\
P_{n}(1)=P_{n-1}(0)+\frac{1}{3} P_{n-1}(2) \\
P_{n}(0)=\frac{1}{2} P_{n-1}(1)
\end{array}\right\}
$$

and, for $n=0$,

$$
P_{0}(r)=\delta_{r 0}
$$

We are particularly interested in the mean end-to-end distance of an $n$-step walk,

$$
r_{n}=\sum_{r=0}^{\infty} r P_{n}(r)
$$

Our main result is the following.

THEOREM. The mean end-to-end distance, $r_{n}$, of the random walk defined by (2.2) and (2.3) has the asymptotic form

$$
r_{n} \sim n-2 \log n \text { as } n \rightarrow \infty \text {. }
$$

This result is to be compared with $r_{n}=n$ for the one-sided self-avoiding walker.

\section{Generating functions}

In order to establish our main result (2.5) we first introduce the generating function

$$
G(r, z)=\sum_{n=0}^{\infty} z^{n} P_{n}(r)
$$


Multiplying equations (2.2) and (2.3) by $z^{n}$ and summing on $n$ we immediately obtain

and

$$
\left.\begin{array}{rl}
z^{-1} G(r, z) & =\left(1+\frac{\delta_{r 1}-1}{r}\right) G(r-1, z)+\frac{1}{r+2} G(r+1, z), \quad r \geqslant 1 \\
G(0, z) & =\frac{z}{2} G(1, z)+1 .
\end{array}\right\}
$$

These recursion relations are only marginally easier to handle than the relations (2.2) but various precise statements can be made about them. For our present purposes we need only the following:

Lemma.

$$
\lim _{z \rightarrow 1-} G(0, z) \leqslant 1+\sqrt{ } 3 \text {. }
$$

This result and other properties of the $G(r, z)$ are established in the Appendix.

It is perhaps of interest to note here in passing that for the self-avoiding walk, $G(0, z)$ is trivially unity, where as for the one-sided ordinary walk with neighbouring step probability $\frac{1}{2}$ away from the origin,

$$
G(0, z)=\left(1-z^{2}\right)^{-t}
$$

The above Lemma is also somewhat surprising since from (3.1) and conservation of probability alone

$$
\sum_{r=0}^{\infty} G(r, z)=(1-z)^{-1}
$$

To proceed with the proof of the Theorem, we next define a generating function for $G(r, z)$ by

$$
F(x, z)=\sum_{r=0}^{\infty}(r+1)^{-1} x^{r+1} G(r, z) .
$$

Multiplying (3.2) by $x^{r}$ and summing on $r$, noting that

$$
\frac{\partial F}{\partial x}=\sum_{r=0}^{\infty} x^{r} G(r, z)
$$

we obtain finally the following differential equation for $F(x, z)$ :

$$
(1-z x) \frac{\partial F}{\partial x}+z\left(1-\frac{1}{x^{2}}\right) F=1+z\left(x-\frac{1}{x}\right) G(0, z) \text {. }
$$

In order to calculate the mean end-to-end distance, $r_{n}$, we note from (2.4), (3.1) and (3.6) that

$$
F_{x x}(1, z)=\left.\frac{\partial^{2} F}{\partial x^{2}}\right|_{x=1}=\sum_{n=0}^{\infty} r_{n} z^{n}
$$

so that the asymptotic form of $r_{n}$ as $n \rightarrow \infty$ is determined from the asymptotic form of (3.9) as $z \rightarrow 1-$. 
Very roughly, one can see from (3.8) that as $x \rightarrow 1$

$$
(1-z x) \frac{\partial F}{\partial x} \sim 1
$$

and hence $F_{x x}(1, z) \sim z(1-z)^{-2}$ as $z \rightarrow 1-$, from which it follows that $r_{n} \sim n$ as $n \rightarrow \infty$.

The differential equation (3.8), however, is an elementary first-order linear equation, and since from (3.6) we require $F(x, z)$ to be analytic in a neighbourhood of the origin $x=0$ for (all) fixed $|z|<1$, the appropriate solution is

$$
F(x, z)=[f(x)]^{-1} \int\left\{1+z\left(x-\frac{1}{x}\right) G(0, z)\right\} f(x)(1-z x)^{-1} d x,
$$

where the integrating factor $f(x)$ is given by

$$
f(x)=(1-z x)^{z^{2}-1} x^{-z^{2}} \exp (z / x) .
$$

In principle, (3.11) represents an exact solution to the problem. In practice, of course, the expression (3.11) is extremely unwieldy. It is, nevertheless, ideally suited to asymptotic analysis. Thus, guided by the intuitive result (3.10), we integrate (3.11) by parts to obtain

$$
\begin{aligned}
F(x, z)= & -[z f(x)]^{-1} \int\left\{1+z\left(x-\frac{1}{x}\right) G(0, z)\right\} f(x) \frac{d}{d x} \log (1-z x) d x \\
= & -z^{-1}\left\{1+z\left(x-\frac{1}{x}\right) G(0, z)\right\} \log (1-z x) \\
& +[z f(x)]^{-1} \int\left\{z\left(1+x^{-2}\right) G(0, z)+\left[z\left(1-z^{2}\right)(1-2 x)^{-1}-z x^{-2}-z^{2} x^{-1}\right]\right. \\
& \left.\times\left[1+z\left(x-\frac{1}{x}\right) G(0, z)\right]\right\} f(x) \log (1-z x) d x
\end{aligned}
$$

Writing $f(x)=-z^{-1}(1-z x) f(x)(d / d x) \log (1-z x)$ in the integral appearing in (3.13) and integrating by parts once more we obtain terms of order

$$
\left(1-z^{2}\right)[\log (1-z x)]^{2} \text { and }(1-z x)[\log (1-z x)]^{2} \text {. }
$$

It follows then to leading order in $(1-z)$ that

$$
F(1, z) \sim-\log (1-z) \text { as } x \rightarrow 1-\text {. }
$$

To determine $F_{x x}(1, z)$ for use in (3.9), we divide (3.8) through by $(1-z x)$ and differentiate with respect to $x$ to obtain

$$
\begin{aligned}
F_{x x}(1, z) & =z(1-z)^{-2}+2 z(1-z)^{-1}\{G(0, z)-F(1, z)\} \\
& \sim z(1-z)^{-2}+2 z(1-z)^{-1} \log (1-z) \quad \text { as } z \rightarrow 1-
\end{aligned}
$$

where, in the second step, we have made use of our Lemma. 
The theorem is finally established by expanding the right-hand side of (3.15) and using the result

$$
\sum_{k=1}^{n} k^{-1} \sim \log n \quad \text { as } n \rightarrow \infty
$$

\section{Appendix}

In order to establish the Lemma we first re-express (3.2) for $r \geqslant 2$ as

$$
\frac{G(r, z)}{z^{-1} G(r, z)-[1 /(r+2)] G(r+1, z)}=\frac{G(r, z)}{[1-(1 / r)] G(r-1, z)} .
$$

Defining

$$
A(r)=G(r, z) / G(r-1, z)
$$

we then have

$$
\begin{array}{r}
A(r)=\frac{\alpha_{r}}{1-\beta_{r} A(r+1)} \\
=\frac{\alpha_{r}}{1-\frac{\beta_{r} \alpha_{r+1}}{1-\frac{\beta_{r+1}}{\alpha_{r+2}}}}, \\
1-\ldots
\end{array}
$$

where

$$
\alpha_{r}=z\left(1-\frac{1}{r}\right), \quad \beta_{r}=\frac{z}{r+2}
$$

Now since

$$
\beta_{r+p} \alpha_{r+p+1} \leqslant \beta_{r} \alpha_{r+1} \text { for } r, p \geqslant 1
$$

it follows from (A3) that

$$
A(r) / \alpha_{r} \leqslant \frac{1}{1-\frac{\beta_{r} \alpha_{r+1}}{1-\frac{\beta_{r} \alpha_{r+1}}{1-\ldots}}}=\frac{1}{2 \beta_{r} \alpha_{r+1}}\left\{1-\left(1-4 \beta_{r} \alpha_{r+1}\right)^{\frac{1}{2}}\right\}
$$

In particular when $r=2$, (A2) and (A6) yield

$$
\frac{G(2, z)}{G(1, z)} \leqslant \frac{3}{2 z}\left\{1-\left(1-2 z^{2} / 3\right)^{\frac{1}{2}}\right\}
$$

By definition (3.1), $G(r, z)$ is finite and non-zero in the interval $0<z<1$ so that on multiplying (A7) through by $G(1, z)$ and substituting into (3.2) for $r=1$ we then have (for $0<z<1$ ), 


$$
z^{-1} G(1, z) \leqslant G(0, z)+(2 z)^{-1}\left\{1-\left(1-2 z^{2} / 3\right)^{\frac{1}{2}}\right\} G(1, z) .
$$

Substituting the resulting inequality for $G(1, z)$ into (3.2) with $r=0$ then gives

$$
G(0, z) \leqslant\left\{1+\left(1-2 z^{2} / 3\right)^{\frac{1}{2}}\right\}\left\{1-z^{2}+\left(1-2 z^{2} / 3\right)^{\frac{1}{2}}\right\}^{-1}
$$

which is certainly valid for $0<z<1$. The Lemma then follows from (A9) by taking the limit $z \rightarrow 1-$.

\section{References}

[1] C. Domb, Adv. Chem. Phys. 15 (1969), 229.

[2] S. F. Edwards, Proc. Phys. Soc. 85 (1965), 613.

[3] P. Flory, J. Chem. Phys. 17 (1949), 303.

[4] P. de Gennes, Rep. Prog. Phys. 32 (1969), 187.

[5] F. T. Wall and J. J. Erpenbeck, J. Chem. Phys. 30 (1959), 634.

Department of Physics

University of Melbourne

Parkville, Vic. 3052

and

Department of Mathematics

University of Melbourne

Parkville, Vic. 3052 